\title{
Isoreticular homochiral porous metal-organic structures with tunable pore size
}

\author{
Danil N. Dybtsev, ${ }^{a, b}$ Maxim P. Yutkin, ${ }^{a}$ Eugenia V. Peresypkina, ${ }^{a}$ Alexander V. Virovets, ${ }^{a}$ \\ Christian Serre, ${ }^{b}$ Gérar Férey, ${ }^{b}$ Vladimir P. Fedin* ${ }^{*}, a$ \\ $a=$ Nikolaev Institute of Inorganic Chemistry, Siberian Branch of the Russian Academy of Sciences. Pr. Lavrentieva \\ 3, Novosibirsk630090, Russia. E-mail: cluster@che.nsk.su \\ $b=$ Institut Lavoisier, Université de Versailles St-Quentin en Yvelines, 45 Avenue des Etats- \\ Unis, 78035 Versailles Cedex, France.
}

\section{SUPLEMENTARY INFORMATION}

Starting materials, instruments and methods. The starting compounds, viz., zinc nitrate (analytical grade), (+)-camphoric acid $\left(\mathrm{H}_{2} \mathrm{camph}\right.$, Aldrich), diazabicyclo[2.2.2]octane (dabco, Aldrich), 4,4'-bipyridyl (bipy, Aldrich), trans-bis(4-pyridyl)ethylene (bpe, Aldrich) were used without additional purification. The other reagents and solvents were of reagent grade. The elemental analysis was carried out in the Laboratory of Microanalysis of the Vorozhtsov Institute of Organic Chemistry (Novosibirsk, Russia). The single crystal X-ray analyses were carried out on the Bruker Nonius X8Apex diffractometer, equipped with 4K CCD detecter. The powder diffraction data were collected using DRON-SEIFERT-RM4 and Siemens D5000 diffractometers $(\lambda=1.54056)$. The IR spectra in $\mathrm{KBr}$ pellets were recorded on Scimitar FTS 2000 and Nicolet-Magma IR550 instruments. The TGA plots were recorded on the TAInstruments TGA-2050 apparatus in $\mathrm{N}_{2}$ atmosphere at $10 \mathrm{deg} / \mathrm{min}$ heating rate. The $\mathrm{N}_{2}$ sorption measurements for 1-3 were performed on a Micromeritics ASAP 2010 at $77 \mathrm{~K}$.

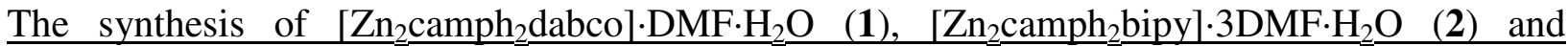

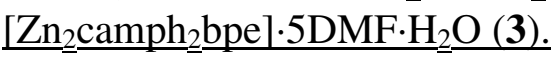

The same synthetic method was carried out for all the compounds. The mixture of $\mathrm{Zn}\left(\mathrm{NO}_{3}\right)_{2} \cdot 6 \mathrm{H}_{2} \mathrm{O}(150 \mathrm{mg}, 0.5 \mathrm{mmol})$, (+)- $\mathrm{H}_{2} \mathrm{camph}(100 \mathrm{mg}, 0.5 \mathrm{mmol})$ and $0.25 \mathrm{mmol}$ of corresponding $\mathrm{N}$-donor ligand (28 mg for dabco; $39 \mathrm{mg}$ for bipy; $46 \mathrm{mg}$ for bpe) in $5 \mathrm{ml}$ of $\mathrm{N}, \mathrm{N}^{\prime}$-dimethylformamide (DMF) was heated in the screwed-cap teflone vial at $110{ }^{\circ} \mathrm{C}$ for 2 days. The block-shaped crystals were collected, washed with DMF and dried on air. Yield: 100 $\mathrm{mg}$ for $\mathbf{1}(55 \%), 180 \mathrm{mg}$ for $\mathbf{2}(78 \%), 182 \mathrm{mg}$ for $\mathbf{3}(67 \%)$. Elemental analysis. Found for 1: C, 46.51; H, 6.51; N, 5.98. Calculated for $\left[\mathrm{Zn}_{2}\left(\mathrm{C}_{10} \mathrm{H}_{14} \mathrm{O}_{4}\right)_{2}\left(\mathrm{C}_{6} \mathrm{H}_{12} \mathrm{~N}_{2}\right)\right] \cdot \mathrm{DMF} \cdot 1.7 \mathrm{H}_{2} \mathrm{O}: \mathrm{C}, 46.87 ; \mathrm{H}$, 6.84; N, 5.65. Found for 2: C, 50.77; H, 6.61; N, 7.60. Calculated for $\left[\mathrm{Zn}_{2}\left(\mathrm{C}_{10} \mathrm{H}_{14} \mathrm{O}_{4}\right)_{2}\left(\mathrm{C}_{10} \mathrm{H}_{8} \mathrm{~N}_{2}\right)\right] \cdot 3 \mathrm{DMF} \cdot \mathrm{H}_{2} \mathrm{O}: \mathrm{C}, 50.88 ; \mathrm{H}, 6.46 ; \mathrm{N}, 7.61$. Found for 3: C, 49.77; H, 6.59; N, 8.23. Calculated for $\left[\mathrm{Zn}_{2}\left(\mathrm{C}_{10} \mathrm{H}_{14} \mathrm{O}_{4}\right)_{2}\left(\mathrm{C}_{12} \mathrm{H}_{10} \mathrm{~N}_{2}\right)\right] \cdot 4.5 \mathrm{DMF} \cdot 3 \mathrm{H}_{2} \mathrm{O}$ : $\mathrm{C}, 50.03 ; \mathrm{H}, 6.97 ; \mathrm{N}$, 8.33. TGA data. Found solvent weight loss for 1: $11 \%\left(12 \%\right.$ calculated for DMF $\left.+\mathrm{H}_{2} \mathrm{O}\right)$. Found solvent weight loss for 2: $24 \%$ ( $26 \%$ calculated for $\left.3 \mathrm{DMF}+\mathrm{H}_{2} \mathrm{O}\right)$. Found solvent weight loss for 3: $36 \%$ (35\% calculated for $5 \mathrm{DMF}+\mathrm{H}_{2} \mathrm{O}$ ). The corresponding TGA curves for as-synthesised samples 1-3 could be seen on Fig. S1. The FT-IR spectra for both the as-synthesized 1-3 and corresponding guest-free samples, heated in vacuo for 1 day at $120{ }^{\circ} \mathrm{C}$, could be found on the Fig. S2. The powder X-ray patterns for 1 and 2, together with the calculated plots (based on the single-crystal X-ray data) are presented on Figs. S3 and S4. 
X-ray single crystal analysis for $\mathbf{1}$ and $\mathbf{2}$.

Diffraction data were collected at $90 \mathrm{~K}$ by standard technique on Bruker X8 Apex CCD diffractometer $\left(\mathrm{MoK}_{\alpha}\right.$ radiation, graphite monochromator). Full sphere in reciprocal space was collected up to $2 \theta=55 \mathrm{deg}$. Absorption correction was applied using SADABS program.

Structure solution and refinement for 1. Structure was refined in mixed isotropic/anisotropic approximation for non-hydrogen atoms: isotropic for carbon atoms of dabco spacer and anisotropic for the rest atoms. In both models dabco spacer is disordered by rotation over $\mathrm{Zn}-\mathrm{N}$ axis. No solvent molecules were located in both models probably due to severe disorder. Hydrogen atoms were not located.

Crystal data for $\mathrm{C}_{2} \underline{\mathrm{H}_{4}} \underline{\mathrm{N}}_{2} \underline{\mathrm{N}}_{2} \underline{\mathrm{O}}_{8} \underline{\mathrm{Zn}}_{2}$ (1 without solvent molecules): No solvent molecules were located probably due to severe disorder. $M=719.34$, tetragonal, space group $P 42_{1} 2$ (No. 90), $a$ $=13.4551(2) \AA, c=9.5832(2) \AA, V=1734.94(5) \mathrm{A}^{3}, Z=2, D_{\mathrm{c}}=1.377 \mathrm{~g} / \mathrm{cm}^{3}, F 000=748$, Mo $\mathrm{K} \alpha$ radiation, $\lambda=0.71073 \mathrm{~A}, T=90.0(2) \mathrm{K}, 2 \theta_{\max }=55.0^{\circ}, 8664$ reflections collected, 2008 unique $\left(R_{\text {int }}=0.0359\right)$; final $\mathrm{GOF}=1.165, \mathrm{R}_{1}=0.0489, w \mathrm{R}_{2}=0.1974, R$ indices based on 1792 reflections with $I>2 \sigma(I)$ (refinement on $F^{2}$ ), 121 parameters, 0 restraints; absorption corrections applied, $\mu=1.442 \mathrm{~mm}^{-1}$. Flack parameter $=0.01(4)$.

Unit cell, space group choice and models for $\mathbf{2}$. The diffraction pattern for $\mathbf{2}$ has pronounced tetragonal symmetry. The unit cell parameters refinement leads to $a$ value undistinguishable from $b$ within experimental precision. The lattice extinctions analysis in tetragonal body centered unit cell shows that glide plane $c$ is present: only 81 of $473 \mathrm{okl}(\mathrm{hol})$ reflections with $l=2 n+1$ has the intensity $\mathrm{I}>3 \sigma(\mathrm{I})$, average $\mathrm{I} / \sigma$ of the reflections to be absent is 1.9 . At that $\mathrm{I} / \sigma$ magnitude for some reflections to be absent are of 30 . Thus, the overall diffraction pattern for $\mathbf{I}$ is in a good agreement with $I 4 c 2$ space group. However, this space group contradicts to the fact of presence of optically pure camphoric acid in the structure. In fact, the refinement of the structure in $I 4 c 2$ space group does not allow localizing position of the methyl group at chiral centre of camphoric acid. At the same time the molecule is disordered by $180^{\circ}$ rotation around two-fold axis parallel to $a$ or $b$ (Fig.1). Taking into account the contradiction between inversion axis of the space group found, on the one hand, and, chirality of the crystal, on the other, we supposed that $I 4 c 2$ space group corresponds to idealized substructure. Proper space group must be maximal chiral subgroup of $I 4 c 2$. Because there is no reason to doubt in the length of primitive translations it must be $t$-subgroup (translationengleiche). According to International Tables for Crystallography orthorhombic $F 222$ space group is one of the maximal non-isomorphic $t$ subgroups of $I 4 c 2$, together with $I 4$ and $I \mathrm{ba} 2$, and the only chiral one. Therefore $F 222$ only obey simultaneously both conditions. Switching to $F 222$ results in doubled asymmetric unit and doubled cell volume, but both crystallographically independent camphoric acid molecules are still disordered. Structure refinement in F222 space group met a number of difficulties. First, $\mathrm{R}_{\text {int }}$ in $14 c 2$ space group is quite low (0.034), i.e. the crystal seems to be tertragonal not only metrically, but the intensities of reflections are also in good agreement with tetragonal Laue class $4 / \mathrm{mmm}$. Thus, $I 4 c 2 \rightarrow F 222$ transition lead to strong pseudosymmetry effects and, therefore, strong correlations between two parts of asymmetric unit. However, carbon atom position of methyl group at chiral centre of camphoric acid can be found from $\Delta \rho$, but geometric constraints have to be applied at refinement. To avoid correlation effect we have to use block diagonal least squares refinement instead of full-matrix one for two parts of asymmetric unit, related by $S_{4}$ pseudo-axis, in different blocks.

Both models presented have their advantages and disadvantages. The $I 4 c 2$ model almost ideally corresponds to symmetry of diffraction pattern observed and obey the principle of minimal crystallographic description. It has no correlations at full-matrix least squares refinement, but 
contradicts to chirality of (+)-camphoric acid. The position of the methyl group at chiral centre of camphoric acid can only be found geometrically, not objectively from $\Delta \rho$. The $F 222$ model, in contrast, allows chirality of camphoric acid. The deviation of the structure from $I 4 c 2$ space group is only due to disordered $\mathrm{CH}_{3}$ group, which contributes a little to the diffraction. It is the methyl group, which apparently is responsible for reflections to be absent in $\mathrm{Okl}(\mathrm{hOl})$. However, if we reject glide plane and switch to $I 4$ space group the problem of chirality for the camphoric acid molecule cannot be resolved. This is the reason to perform the next step and to remove $S_{4}$ inversion axis that means to leave tetragonal symmetry and switch to $F 222$ space group.

Structure solution and refinement for $\mathbf{2}$. Tetragonal centrosymmetric model of the structure (in $I 4 c 2$ space group) was found by direct methods using XS program of SHELXTL 6 program package. Model in F222 space group was generated from centrosymmetric one mathematically by reducing the symmetry and transformation of the unit cell. In both models structure was refined in mixed isotropic/anisotropic approximation: isotropic for carbon atoms of bipy spacer and anisotropic for the rest atoms. In both models bipy spacer is disordered over two orientations $(0.55 / 0.45)$ by rotation over $\mathrm{Zn}-\mathrm{N}$ axis. The refinement of centrosymmetric model was fullmatrix while the non-centrosymmetric model required block-diagonal one to avoid correlation effect. Hydrogen atoms were not located. Due to the pseudo-centosymmetric nature the parameter of absolute structure (Flack parameter) was impossible to be refined properly. No solvent molecules were located in both models probably due to severe disorder.

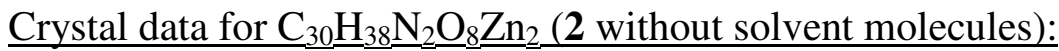

Non-centrosymmetric model for 2. $M=685.36$, orthorhombic, space group $F 222$ (No. 22), $a=$ 18.8703(3) $\AA, b=18.8703(3) \AA, c=27.8994(12) \AA, V=9934.6(5) \mathrm{A}^{3}, Z=8, D_{\mathrm{c}}=0.916 \mathrm{~g} / \mathrm{cm}^{3}$, $F 000=2848$, Mo K $\alpha$ radiation, $\lambda=0.71073 \mathrm{~A}, T=90.0(2) \mathrm{K}, 2 \theta_{\max }=55.0^{\circ}, 8893$ reflections collected, 4787 unique $\left(R_{\text {int }}=0.0293\right)$; final $\mathrm{GOF}=1.494, \mathrm{R}_{1}=0.0724, w \mathrm{R}_{2}=0.2170, R$ indices based on 2802 reflections with $I>2 \sigma(I)$ (refinement on $F^{2}$ ), 228 parameters, 2 restraints; absorption corrections applied, $\mu=0.998 \mathrm{~mm}^{-1}$. Flack parameter $=0.13(5)$.

Pseudo-centrosymmetric model for 2. $M=685.36$, orthorhombic, space group $I 4 c 2$ (No. 116), $a$ $=13.3433(3) \AA, c=27.8994(12) \AA, V=4967.3(3) \mathrm{A}^{3}, Z=4, D_{\mathrm{c}}=0.914 \mathrm{~g} / \mathrm{cm}^{3}, F 000=1416$, Mo K $\alpha$ radiation, $\lambda=0.71073 \mathrm{~A}, 2 \theta_{\max }=55.0^{\circ}, 8447$ reflections collected, 2830 unique $\left(R_{\text {int }}=\right.$ 0.0340 ); final GOF $=1.190, \mathrm{R}_{1}=0.0755, w \mathrm{R}_{2}=0.2269, R$ indices based on 1770 reflections with $I>2 \sigma(I)$ (refinement on $F^{2}$ ), 119 parameters, 0 restraints; absorption corrections applied, $\mu$ $=0.997 \mathrm{~mm}^{-1}$. 
Structure determination of $\mathbf{3}$ from powder X-ray data. Our attempts to index the unit cell from the X-ray powder diffractogram of $\mathbf{3}$ failed, probably due to its irregular crystal structure. Therefore, the simulated X-ray patterns for $\mathbf{3}$ was calculated, ${ }^{\mathrm{REF}}$ assuming the same $\left\{\mathrm{Zn}_{2} \mathrm{Camph}_{2}\right\}$ layered motif as $\mathbf{1}$ and $\mathbf{2}$, pillared by bpe ligands. Two possibilities of layer packing (AAAA and $\mathrm{ABAB}$ ) were taken into account. The $a$ and $c$ parameters of the (pseudo)tetragonal unit cell were refined from the positions of corresponding reflexes and were found to be $a=13.533 \AA, c=16.265 \AA$ for primitive unit cell $\mathrm{P} 42_{1} 2$ (AAAA packing mode) and $a=b=19.139 \AA, c=32.530 \AA$ for the face-centered unit cell $F 222$ (ABAB packing mode). Thus, the found parameters $a$ and $b$ in $\mathbf{3}$ are similar to those in $\mathbf{1}$ and $\mathbf{2}$, while some increase of parameter $c$ is in the perfect correlation with the proposed structural features of $\mathbf{3}$ and the elongation of the interlayer distances due to bpe ligand. The experimental X-ray powder diffraction pattern for $\mathbf{3}$ together with calculated ones are shown on Fig. S5.

Framework stability of host structures 1-3 after high-temperature guest removal.

The crystalline samples 1-3 were heated in vacuo for 1 day at $120{ }^{\circ} \mathrm{C}$. The FT-IR spectra of guest-free materials (Fig. S2) show the absence of the guest DMF molecules (sharp peak at $1560-1580 \mathrm{~cm}^{-1}$ ). The stability of the metal-organic host frameworks in $\mathbf{1 - 3}$ after that guest removal was monitored by powder X-ray diffraction. The host metal-organic framework in $\mathbf{1}$ seems to retain its structure after the guest removal, while the guest-free frameworks $\mathbf{2}$ and $\mathbf{3}$ probably collapse. After that, the amorphous guest-free samples of $\mathbf{2}$ and $\mathbf{3}$ were soaked in DMF for 1 week at room temp. The X-ray powder diffraction patterns of the corresponding samples show, that framework in $\mathbf{2}$ restores its crystallinity, but the sample $\mathbf{3}$, with the longest bpe linker does not. The corresponding X-ray patterns could be found on Fig. S6.

The porosity of the guest-free evacuated samples 1-3 was additionally checked by the $\mathrm{N}_{2}$ sorption. Similar to the X-ray powder diffraction data, only the evacuated sample 1 possesses the reversible type I sorption isotherm, typical to microporous materials $\left(S_{\text {Langmuir }}=553 \mathrm{~m}^{2} / \mathrm{g}\right)$. No nitrogen sorption was found for the guest-free samples 2 and $\mathbf{3}$. The $\mathrm{N}_{2}$ sorption isotherm for guest-free framework 1, together with Langmuir linear plot could be found on the Fig. S7.

Framework stability in $\mathbf{2}$ and $\mathbf{3}$ upon guest-exchange in mild conditions. The crystalline samples 2 and 3 were soaked in $\mathrm{CH}_{2} \mathrm{Cl}_{2}$ for 1 week at room temperature. During that time the solvent was renewed several times to facilitate complete guest DMF removal from the porous structures. The absence of DMF molecules in guest-exchanged $\mathbf{2}$ and $\mathbf{3}$ was checked from IR spectra (disappearance of strong and sharp $\mathrm{C}=\mathrm{O}$ stretching at $1560-1580 \mathrm{~cm}^{-1}$, compared to assynthesized materials). After that, the DMF-free crystals were soaked in DMF for another week at room temp. The stability of the metal-organic host framework in $\mathbf{2}$ and $\mathbf{3}$ during that guest exchange was monitored by powder X-ray diffraction. The corresponding patterns could be found on Fig. S8. 

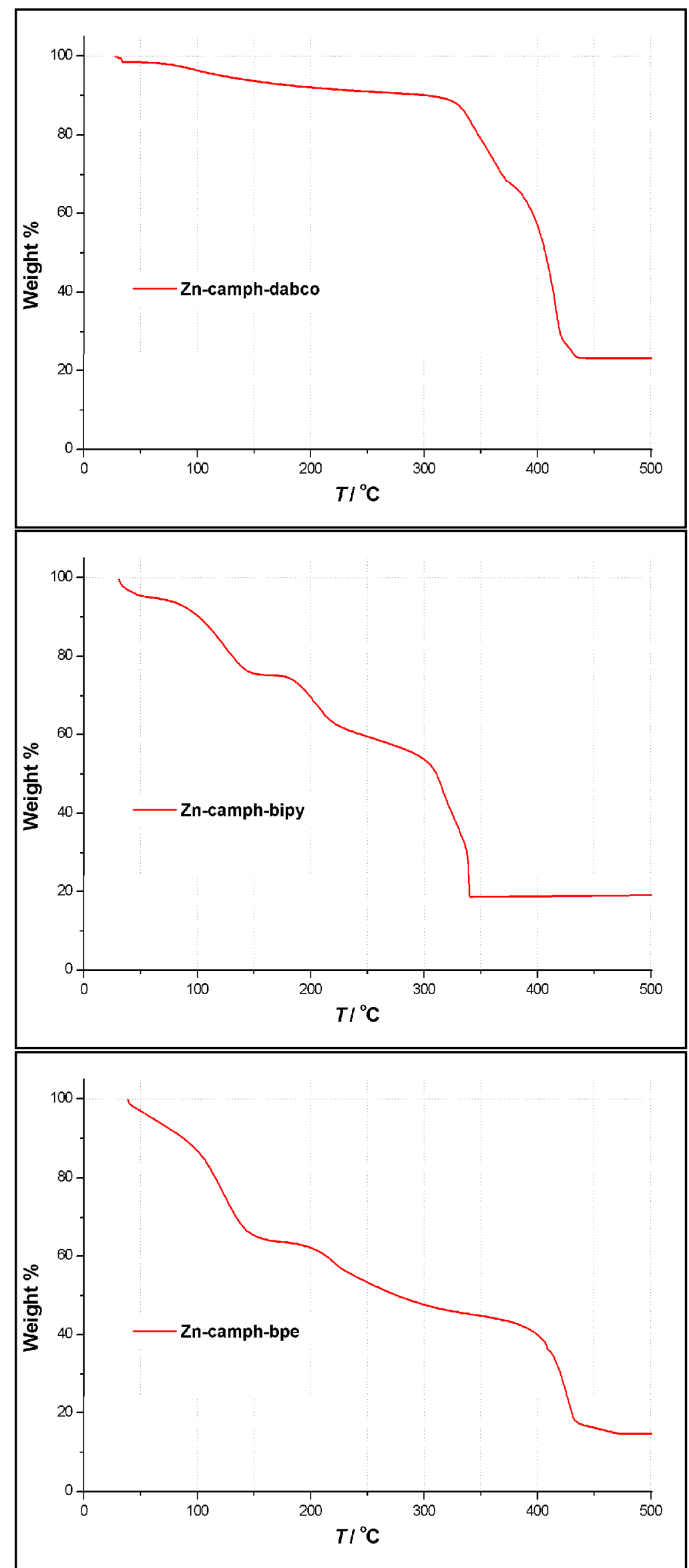

Fig. S1. The TGA plots for the as-synthesized samples $\mathbf{1}$ (top), $\mathbf{2}$ (middle) and $\mathbf{3}$ (bottom). 

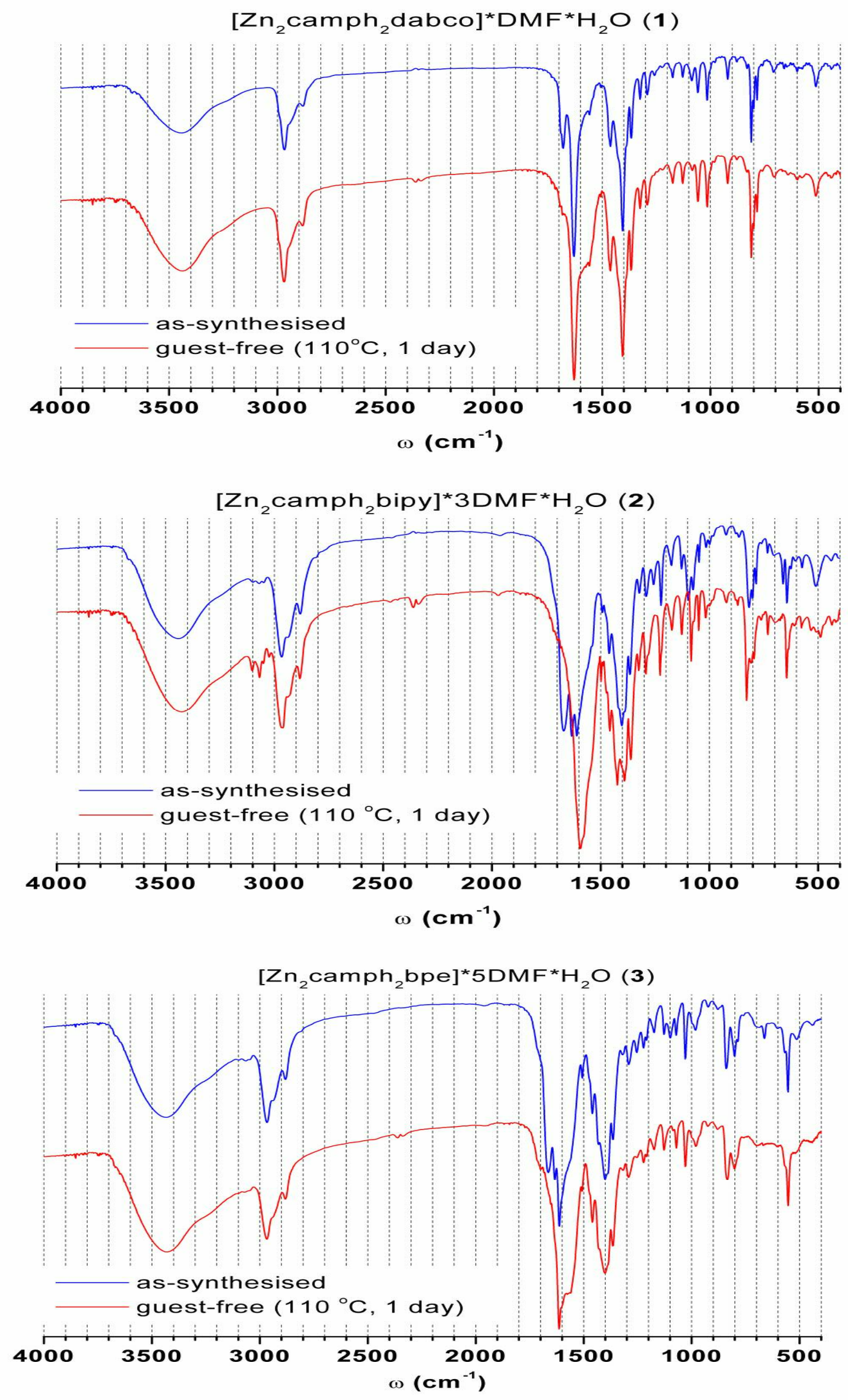

Fig. S2. The FT-IR spectra of the as-synthesised samples $\mathbf{1}$ (top), 2 (middle) and $\mathbf{3}$ (bottom) and the corresponding guest-free frameworks, heated in vacuo at $120{ }^{\circ} \mathrm{C}$ for 1 day. 


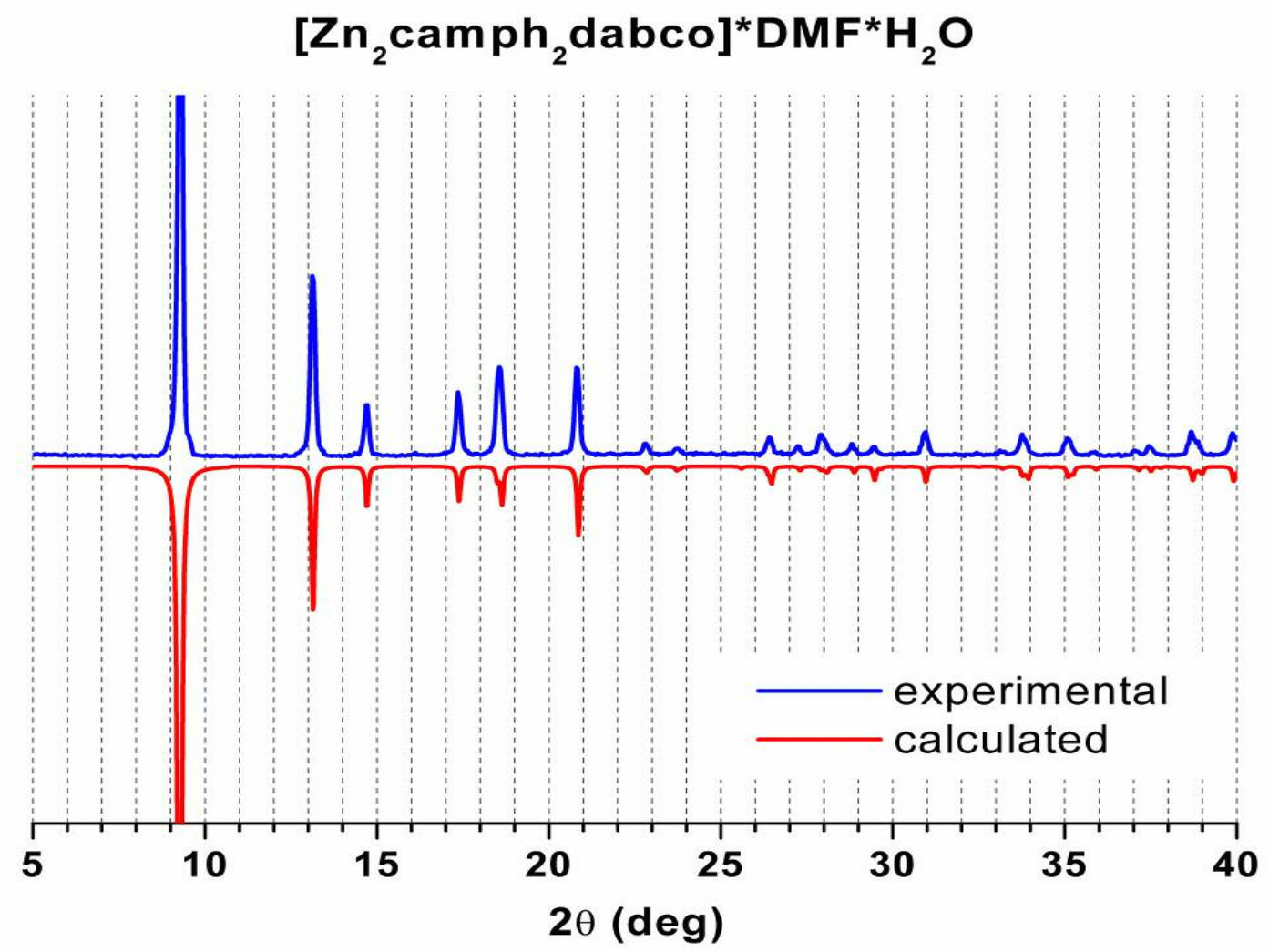

Fig. S3. The experimental powder diffraction pattern of $\mathbf{1}$ (blue) and the corresponding calculated plot (red), based on the single-crystal diffraction data.

\section{$\left[Z n_{2}\right.$ camph $_{2}$ bipy] ${ }^{*}$ DMF $^{*} \mathrm{H}_{\mathbf{2}} \mathbf{O}$}

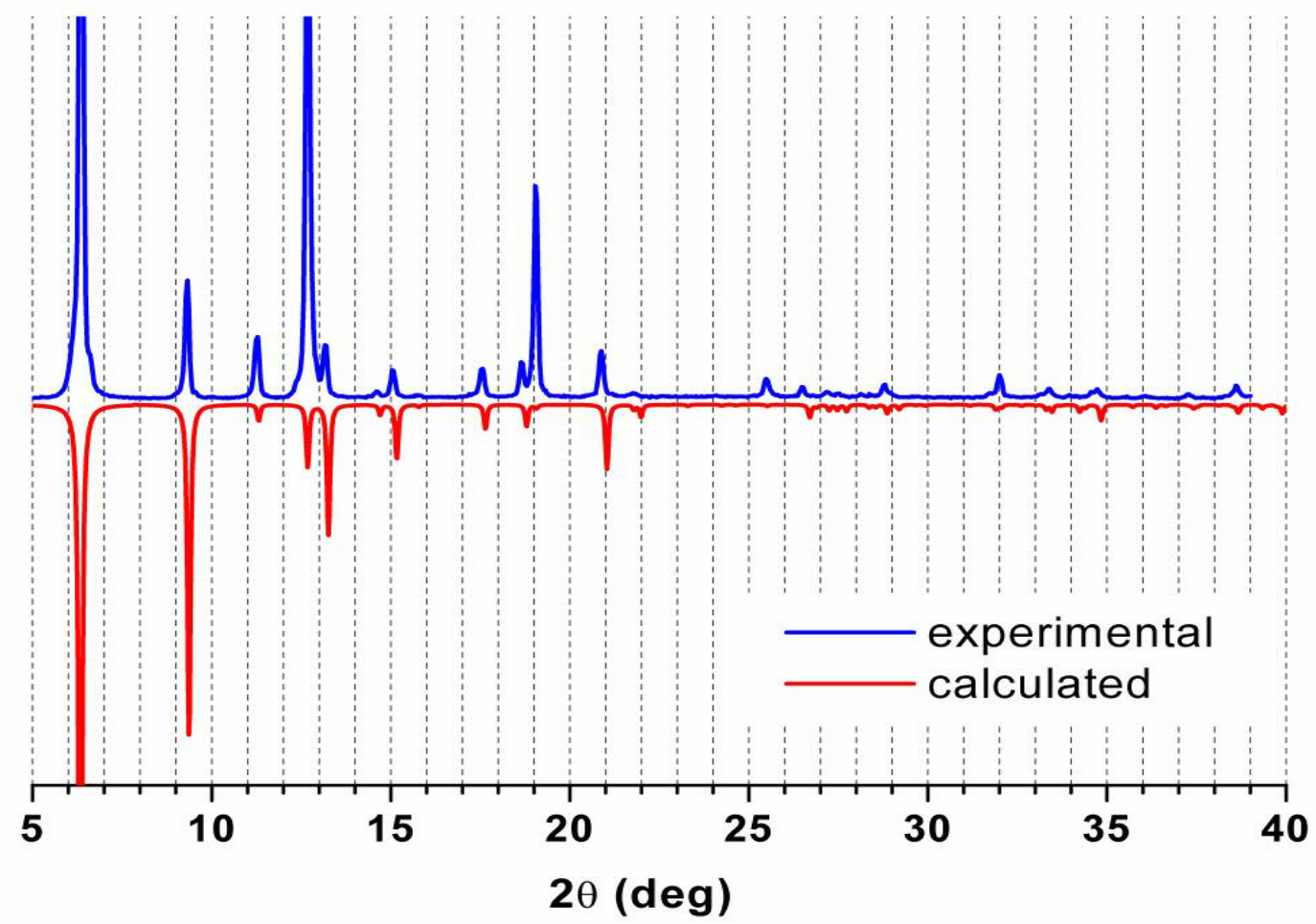

Fig. S4. The experimental powder diffraction pattern of $\mathbf{2}$ (blue) and the corresponding calculated plot (red), based on the single-crystal diffraction data. 


\section{$\left[\mathrm{Zn}_{2} \mathrm{camph}_{2} \mathrm{bpe}\right] \cdot 5 \mathrm{DMF} \cdot \mathrm{H}_{2} \mathrm{O}$}

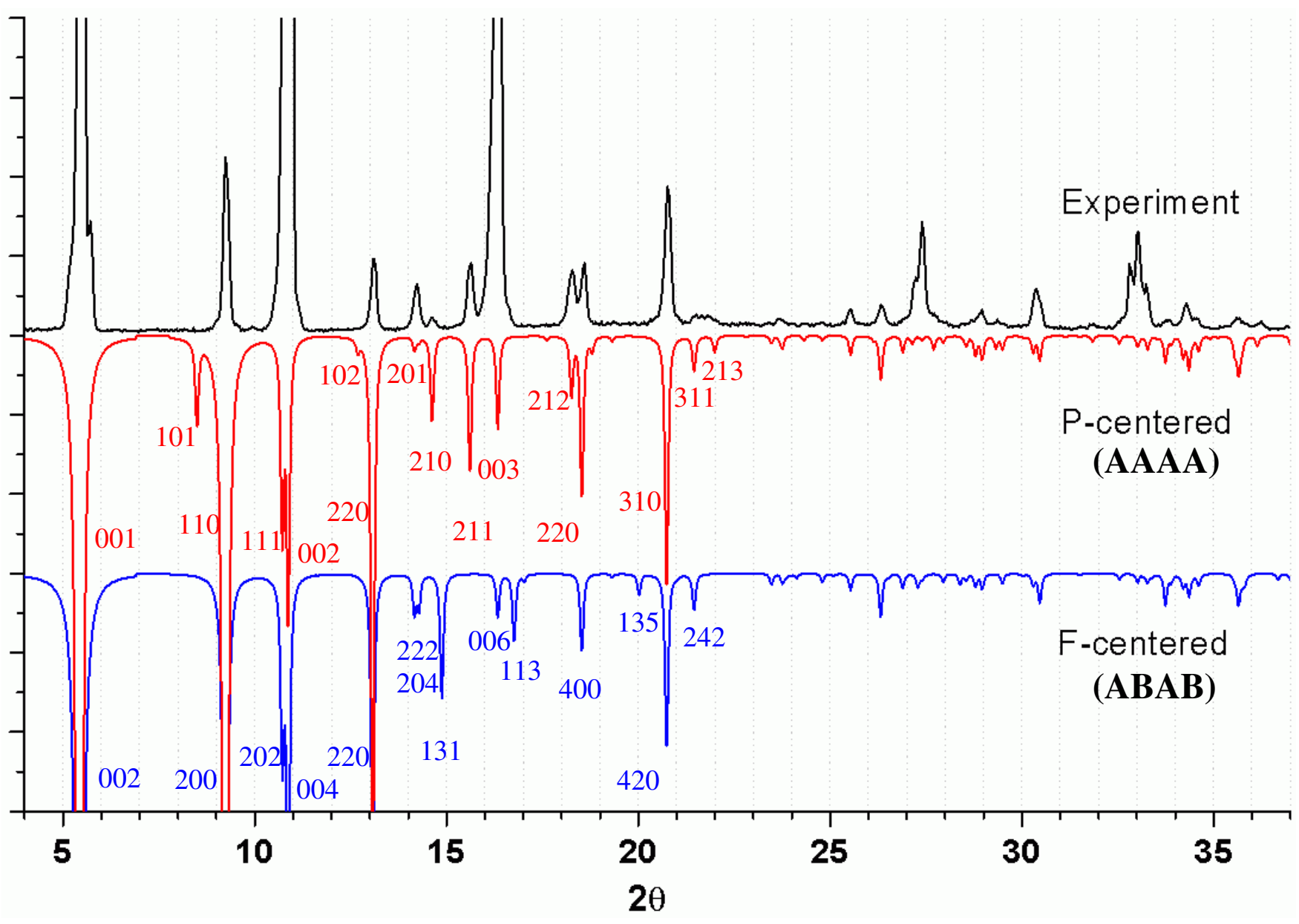

Fig. S5. The experimental X-ray powder diffraction pattern for $\mathbf{3}$ (top, black line) together with calculated ones, assuming primitive unit cell (middle, red line) or face-centered unit cell (bottom, blue line). Some reflections are indexed. 

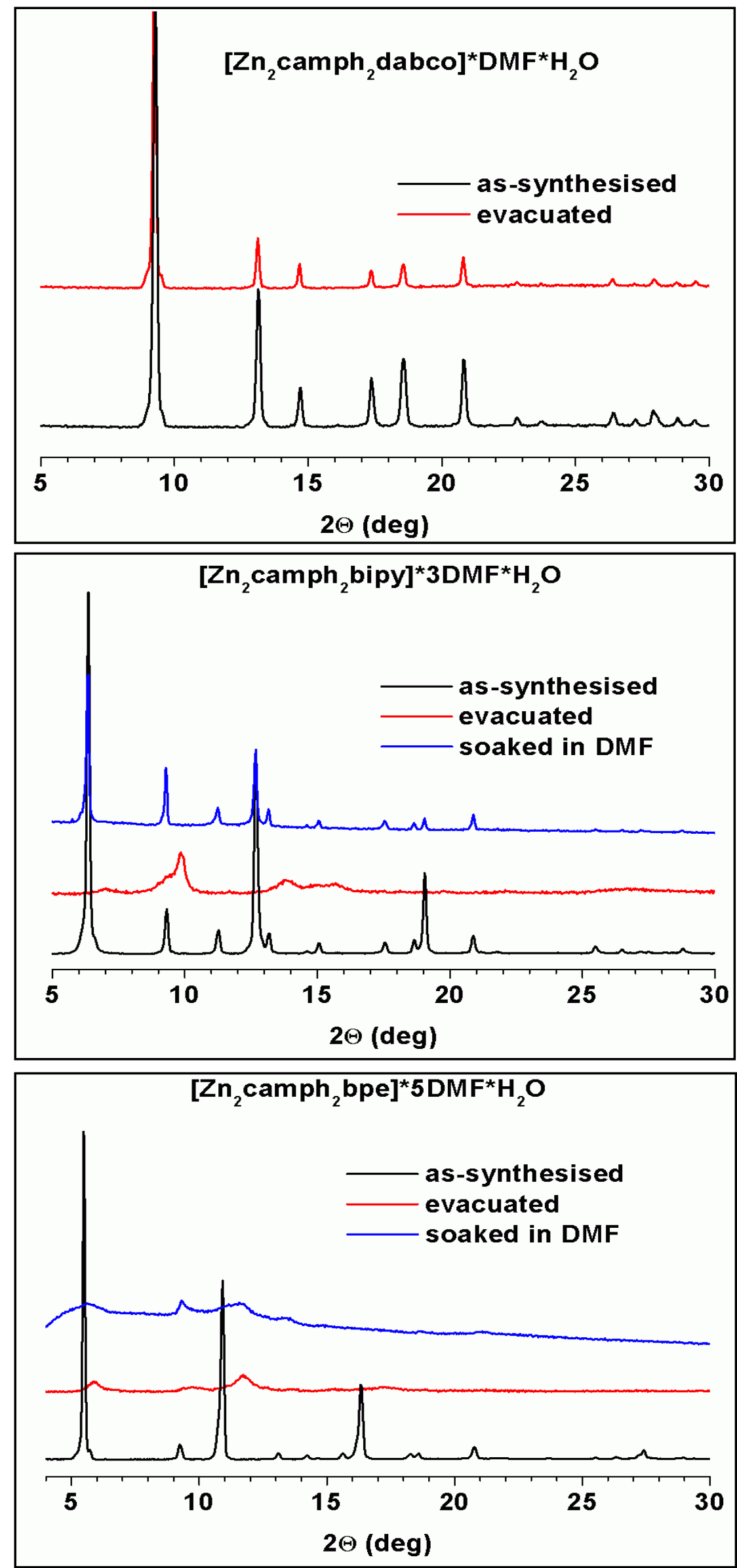

Fig. S6. The experimental X-ray powder diffraction patterns for $\mathbf{1}$ (top), 2, (middle) and $\mathbf{3}$ (bottom) with the corresponding guest-free frameworks (vacuum, $120^{\circ} \mathrm{C}, 1$ day) and after soaking in DMF (for 2 and 3 only) 

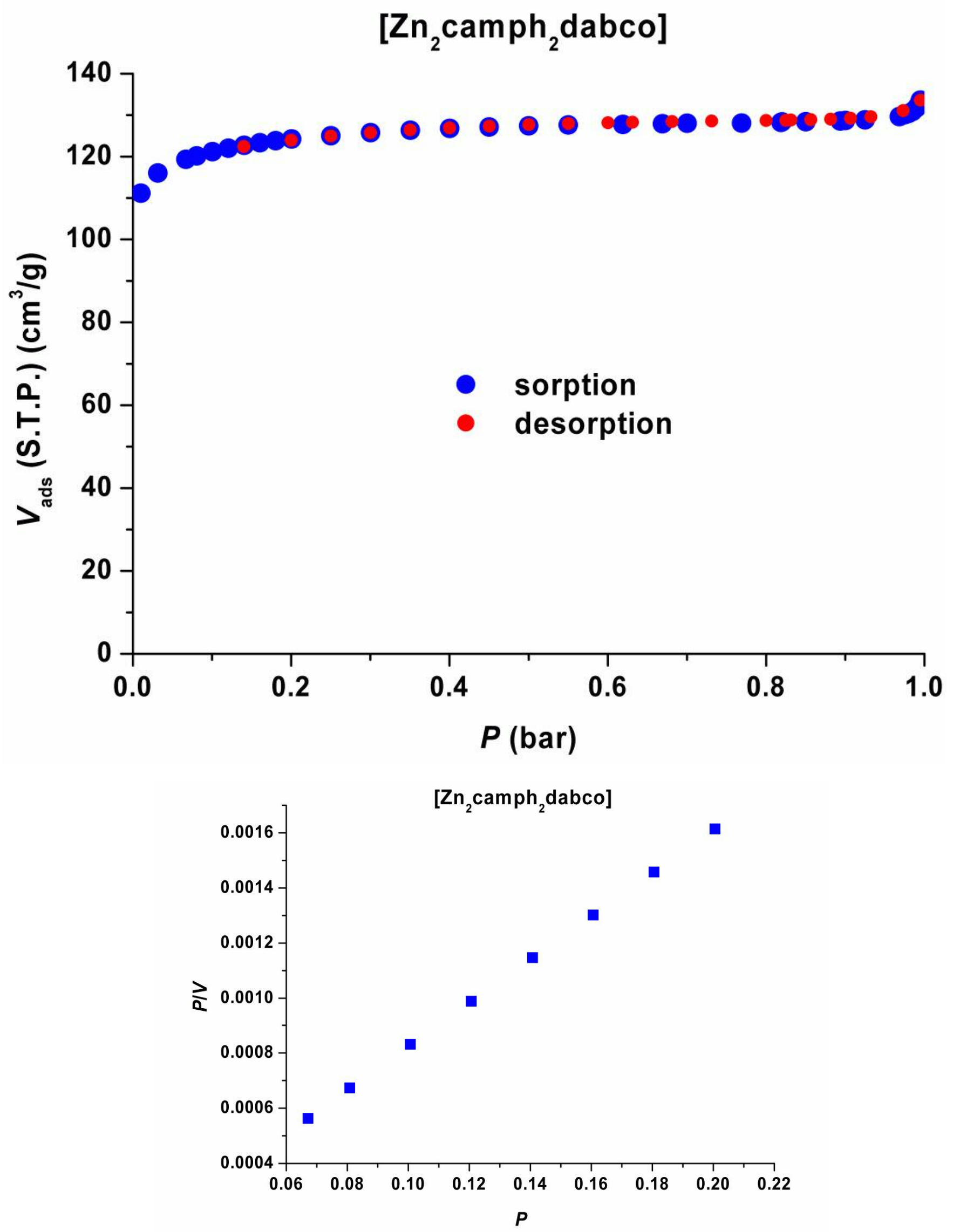

Fig. S7. The nitrogen sorption data at $77 \mathrm{~K}$ for the guest-free framework $\mathbf{1}$ (vacuum, 1 day, $120{ }^{\circ} \mathrm{C}$ ) (top), and corresponding Langmuire plot (bottom). 

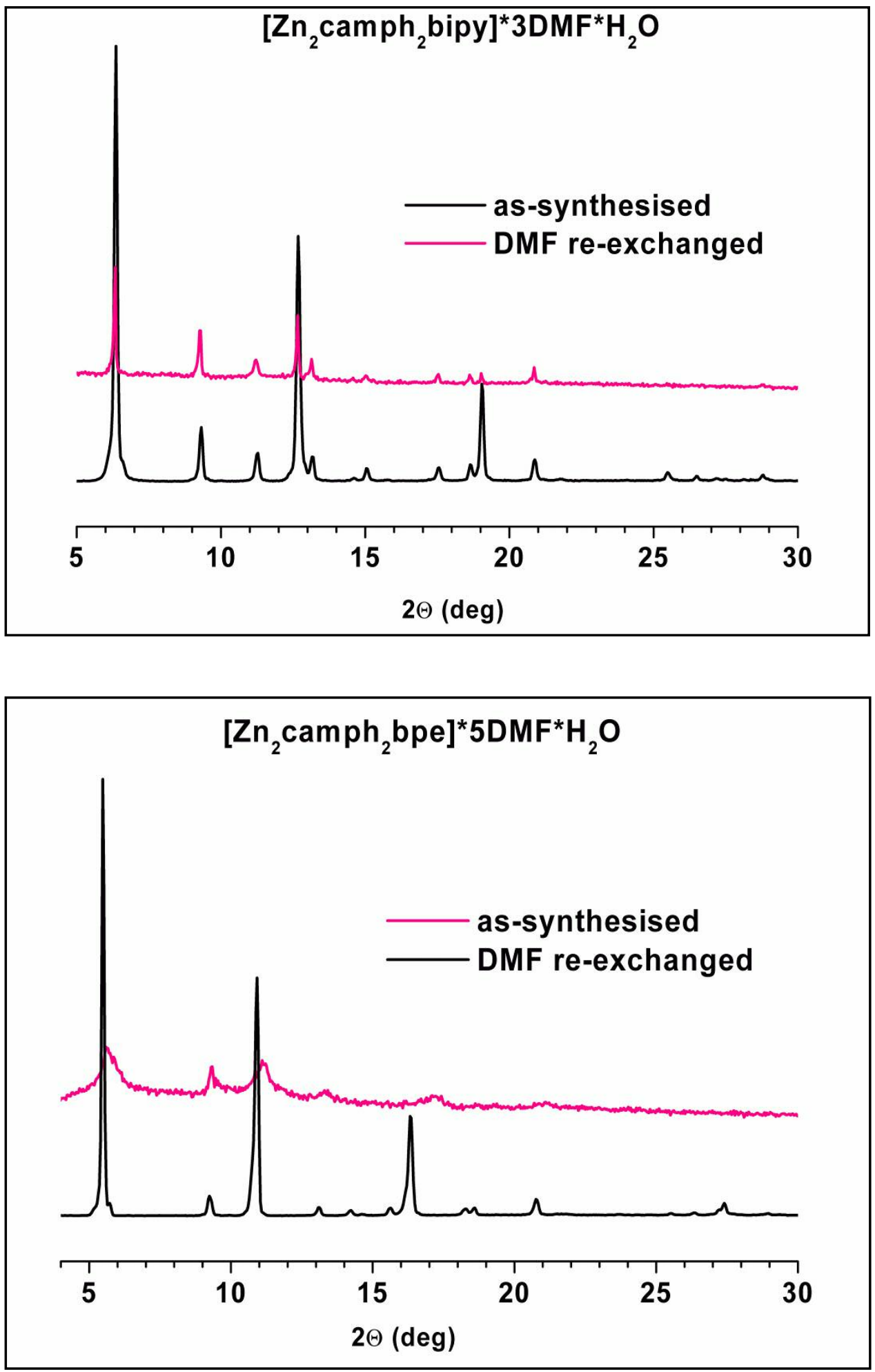

Fig. S8. The experimental X-ray powder diffraction patterns for $\mathbf{2}$ (top) and $\mathbf{3}$ (bottom), showing both assynthesized samples and solvent re-exchanged samples at mild conditions. 